\title{
PARALLEL CONNECTION MEASURING METHOD FOR GAS LEAKAGE BASED ON STANDARD FLOW
}

\author{
Liang HUANG*, Maolin CAI** \\ * Graduate School of Automation Science and Electrical Engineering \\ Beihang University \\ 37 Xueyuan Road, Haidian, Beijing, 100083 China \\ (E-mail: huang1liang2@hotmail.com) \\ ** School of Automation Science and Electrical Engineering \\ Beihang University \\ 37 Xueyuan Road, Haidian, Beijing, 100083 China
}

\begin{abstract}
The objective of work described in this paper is to propose a new method of measuring gas leakage for pneumatic industry. The measurement is enabled by employing standard flow. Standard flow is used to determine the internal volume of the measured equipment. An algorithm is formulated to describe, to the extent possible, the relationship between gas leakage and standard flow. This measurement method deviates from the theoretical leakage values by less than $5 \%$, and shows a good precision and scope compared with the traditional flow measurements. In addition, the proposed parallel connection based on standard flow makes easy operation and fast measuring possible, thus promising new area of application for pneumatic equipments.
\end{abstract}

\section{KEY WORDS}

Gas Leakage, Parallel Connection, Standard Flow

\section{NOMENCLATURE}

$G$ : Massive flow rate

$k$ : Kapper

$m$ : Air mass

$P \quad:$ Internal pressure in pipeline of equipment

$P_{a}:$ Atmospheric pressure

$P_{f}:$ Reference pressure for standard flow

$Q \quad$ : Volumetric flow rate

$R$ : Ideal gas constant

$S_{\mathrm{e}}:$ Effective area

$t$ : Time

$V \quad$ : Internal volume $\theta \quad:$ Air temperature

$\theta_{\alpha}:$ Atmospheric temperature

$\rho:$ : Air density

Suffix:

[kg] $\quad l \quad:$ Leakage without Standard flow

$[\mathrm{Pa}]$

$[\mathrm{Pa}]$

$[1 / \min (\mathrm{ANR})]$

$[\mathrm{J} /(\mathrm{kg} \cdot \mathrm{s})]$

$\left[\mathrm{m}^{2}\right]$

$[\mathrm{s}]$

$\left[\mathrm{m}^{3}\right]$ $s$ : Standard flow

$s l$ : Leakage with Standard flow

\section{INTRODUCTION}

Today, compressed air has been widely used in industrialization since 1980s, because of cleanness, low-cost and easy maintenance of compressed air 
systems[1]. Compressed air systems are consuming approximate 5 percent of the total supplied electricity in china which reaches to two hundred billion $\mathrm{kWh} /$ year. But the consumption of gas leakage takes up to $10-50 \%[2]$. Compared with traditional flow meters, the meter using the proposed new method can be connected parallel to the equipment to detect leakage in the equipment automatically. The relative measurement error can reach to less than $5 \%$. So this method can be largely used for pneumatic equipment.

\section{MEASURING PRINCIPLE}

The Fig. 1 shows the measuring circuit which includes a standard flow circuit.

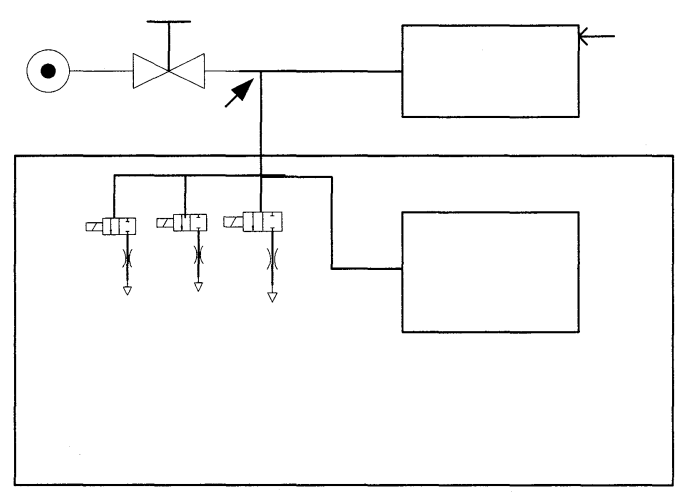

Figure 1 Measuring circuit

Based on thermodynamics, the state equation of compressible air in a chamber can be written as

$$
P V=m R \theta
$$

When leakage happens, the following equation is derived by differentiating Eq.(1):

$V \frac{d P_{l}}{d t}=G_{l} R \theta+m R \frac{d \theta}{d t}$

Where $G$ is negative during discharging, and vice versa. To eliminate the temperature effect on the dynamic air pressure, it is necessary to wait until the temperature in equipment become constant after charging[3][4]. So the temperature in equipment can be considered as atmospheric temperature during the measurement. So the equation obtained from Eq. (2) is

$$
V \frac{d P_{l}}{d t}=G_{l} R \theta_{a}
$$

The volume $\mathrm{V}$ varies from different equipments and pipelines. To void the uncertainty of $\mathrm{V}$, we design a standard flow circuit which includes three solenoid valves. For the volume of solenoid valve is far smaller than that of pipeline in equipment, when the standard flow circuit works, Eq. (3) can be written as

$V \frac{d P_{s l}}{d t}=\left(G_{l}+G_{s}\right) R \theta_{a}$

When leakage happens in the pipeline, compressed air flow through orifice with high speed, the heat can not transfer completely at all.So it can be considered as one-dimensional isentropic flow. For the downstream pressure of leakage passage equals to the atmospheric pressure, the mass flow rate can be expressed as Eq.(5):

$G=S_{e} P B$

Where $\mathrm{B}$ are:

$B=\left\{\begin{array}{c}\sqrt{\frac{\kappa}{R \cdot \theta_{a}}\left(\frac{2}{\kappa+1}\right)^{\frac{\kappa+1}{\kappa-1}}} \quad \frac{P_{a}}{P} \leq 0.5283 \\ \sqrt{\frac{2 \kappa}{(\kappa-1)} \frac{1}{R \cdot \theta_{a}}\left[\left(\frac{P_{a}}{P}\right)^{\frac{2}{\kappa}}-\left(\frac{P_{a}}{P}\right)^{\frac{\kappa+1}{\kappa-1}}\right]} \frac{P_{a}}{P}>0.5283\end{array}\right.$

For $P_{a} / P<0.528$, leakage flow rate is proportional to internal pressure, and we can get the Eq.(6):

$$
\begin{aligned}
& G_{l}=S_{e l} P_{l} \sqrt{\frac{\kappa}{R \cdot \theta_{a}}\left(\frac{2}{\kappa+1}\right)^{\frac{\kappa+1}{\kappa-1}}} \\
& G_{s}=S_{e s} P_{f} \sqrt{\frac{\kappa}{R \cdot \theta_{a}}\left(\frac{2}{\kappa+1}\right)^{\frac{\kappa+1}{\kappa-1}}}
\end{aligned}
$$

In Fig.2, the simulation shows how the internal pressure in equipment pipeline changes during leakage, Where $R$ $=287, \theta=293 \mathrm{k}, V=20 \mathrm{~L}, P_{f}=0.5 M P a, G_{l}=-0.987 \times 10^{-3} \mathrm{~kg} / \mathrm{s}$, $G_{s I}=-0.3957 \times 10^{-3} \mathrm{~kg} / \mathrm{s}, G_{s 2}=-0.987 \times 10^{-3} \mathrm{~kg} / \mathrm{s}, G_{s 3}=$ $-2.0 \times 10^{-3} \mathrm{~kg} / \mathrm{s}$. 


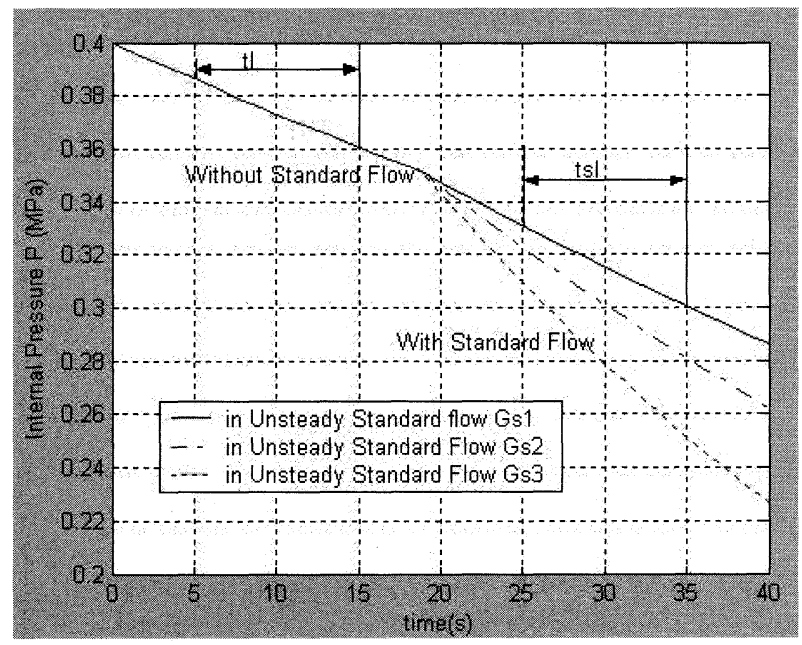

Figure 2 Pressure Change in the Leakage

In the state of leakage, the average internal pressure can be expressed by Eq.(7):

$V \frac{d P_{l}}{d t}=S_{e l} P_{l} R \theta_{a} \sqrt{\frac{\kappa}{R \cdot \theta_{a}}\left(\frac{2}{\kappa+1}\right)^{\frac{\kappa+1}{\kappa-1}}}$

$V \frac{d P_{s l}}{d t}=\left(S_{e l}+S_{e s}\right) P_{s l} R \theta_{a} \sqrt{\frac{\kappa}{R \cdot \theta_{o}}\left(\frac{2}{\kappa+1}\right)^{\frac{\kappa+1}{\kappa-1}}}$

Eq.(7) can be further expressed as Eq.(8) :

$\ln \left(P_{l d} / P_{l b}\right)=S_{e l} R \theta_{a} t_{l} \sqrt{\frac{\kappa}{R \cdot \theta_{a}}\left(\frac{2}{\kappa+1}\right)^{\frac{\kappa+1}{\kappa-1}}}$

$\ln \left(P_{s l d} / P_{s l b}\right)=\left(S_{e l}+S_{e s}\right) R \theta_{a} t_{s l} \sqrt{\frac{\kappa}{R \cdot \theta_{a}}\left(\frac{2}{\kappa+1}\right)^{\frac{\kappa+1}{\kappa-1}}}$

Based on Eq.(6) and Eq.(8), leakage flow rate at $P_{1}$ can be written as Eq.(9):

$$
G_{l}=\frac{G_{s}}{P_{f}} \cdot \frac{P_{l}}{\left(\frac{t_{l}}{t_{s l}} \cdot \frac{\ln \left(P_{s l d} / P_{s l b}\right)}{\ln \left(P_{l d} / P_{l b}\right)}-1\right)}
$$

Where $P_{\text {sld: }}$ Internal pressure during leakage with standard flow at the end time, $P_{s l b}$ : Internal pressure during leakage with standard flow at the beginning time, $P_{l d}$ : Internal pressure during leakage without standard flow at the end time, $P_{l b}$ : Internal pressure during leakage without standard flow at the beginning time.

\section{EXPERIMENT APPARATUS}

The measuring circuit is shown in Fig.3. The pilot valve A and standard vale 1, 2 and 3 are all controlled by PC. The orifice of standard valve is $3 \mathrm{~mm}$, and its maximum pressure can reach to $1.0 \mathrm{MPa}$. The volume of chamber is 30L. The maximum measurement pressure of pressure sensor is $1.0 \mathrm{MPa}$, and the measurement error can reaches to $0.5 \%$. Leakage flow is controlled by a control valve which provides maximum rate $10001 / \min (\mathrm{ANR})$ at $0.6 \mathrm{MPa}$. Real-time pressure signal in the pipeline is gathered by $\mathrm{PC}$. When leakage happens, pressure in equipment will decrease. After standard flow happens, a different differential pressure will occur accordingly. Leakage flow rate can be calculated with two differential pressure changes.

The measuring program is ruled by the following steps:

i) Charge air into the pipeline, and then wait until the temperature recover to the room temperature.

ii) Close the standard flow circuit, then measure the internal pressure in equipment pipeline $P_{l b} 、 P_{l d}$ and leakage period $t_{l}$.

iii) The standard flow circuit will work automatically and measure the internal pressure in equipment pipeline $P_{s l b} 、 P_{s l d}$ and leakage period $t_{s l}$.

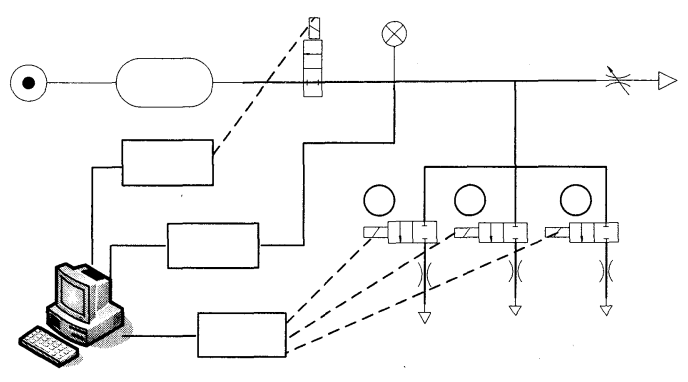

Figure 3 Leakage Flow Measuring Circuit Based on Standard Flow

\section{RESULT AND DISCUSSION}

Based on the measuring principle and experiment apparatus, experiments have been carried out for the different leakage flow. The measurement result is shown in Table.1. For a pressure wave effect to the pressure sensor when standard flow happens, there will be a sudden drop in the pressure curve in figures below. The internal meter pressure data in experiment with sampling frequency $40 \mathrm{HZ}$ are shown in Fig.4 and Fig.5. The Standard leakage flow rate in Fig.4 equals to $-365[1 / \mathrm{min}(\mathrm{ANR})]$ at $0.6 \mathrm{MPa}$, and in Fig.5 equals to $-114[1 / \mathrm{min}(\mathrm{ANR})]$ at $0.6 \mathrm{MPa}$. 


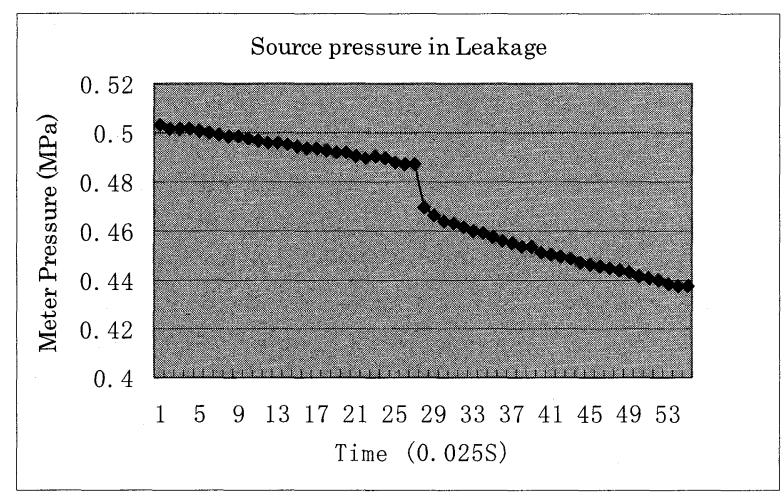

Figure 4 Source pressure in Standard Leakage

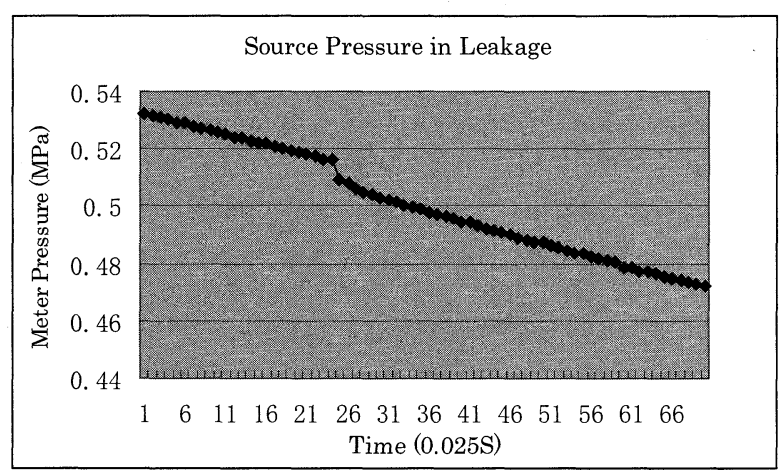

\section{CONCLUSION}

In this paper, a measurement method with parallel connection for gas leakage flow based on standard flow is developed. The relative error has been proved to be less than 5\%. Compared with traditional method, it can be connected to equipment pipeline easily which promises the extensive use in pneumatic industry.

\section{REFERENCES}

1. Maolin Cai, Toshiharu.Kagawa, kenji Kawashima., Energy Conversation and Power Evaluation of Compressible Fluid in Pneumatic Actuator Systems $37^{\text {th }}$ IECEC, 2002, 19-7, pp.438-443

2. Dutch National Team: Compressed air: Saving of $30 \%$ are quite normal, CADDET Energy Efficiency, Newsletter No.3, 1999

3. T.Kato, K.Kawashima, T.Kagawa., Application of Pressure Differential using Isothermal Chamber to Isolation Table, SICE Annual Conference in Sapporo, 2004, 4-8, pp.159-162

4. Ji-Seong Jang, Sang-Won Ji and Bo-Sik kang., Study on the Measurement Method of Leakage Flow-rate for Pnematic Driving Apparatus, SICE-ICASE International Joint Conference in Bexco, Busan, Korea, 2006, 18-10, pp.4116-4120

Figure 5 Source pressure in Standard Leakage

Table 1 Measurement result Table

\begin{tabular}{|c|c|c|c|c|c|}
\hline Leakage No. & $\begin{array}{c}\text { Leakage Flow } \\
{[1 / \mathrm{min}(\mathrm{ANR})]}\end{array}$ & $\begin{array}{c}\text { Standard Flow } \\
{[\mathrm{kg} / \mathrm{s}]}\end{array}$ & $\begin{array}{c}\text { Measure Flow } \\
{[1 / \mathrm{min} \text { (ANR) }]}\end{array}$ & $\begin{array}{c}\text { ERROR } \\
{[\%]}\end{array}$ & $\begin{array}{c}\mathrm{P}_{\mathrm{f}} \\
{[\mathrm{MPa}]}\end{array}$ \\
\hline N0.1 & -460 & -365 & -440.532 & 4.23 & 0.6 \\
\hline N0.2 & -210 & -365 & -217.692 & 3.66 & 0.6 \\
\hline No.3 & -80 & -114 & -77.2415 & 3.44 & 0.6 \\
\hline No.4 & -60 & -114 & -61.007 & 1.66 & 0.6 \\
\hline No.5 & -36 & -114 & -35.347 & 1.81 & 0.6 \\
\hline No.6 & -20 & -114 & -19.380 & 3.10 & 0.6 \\
\hline
\end{tabular}

